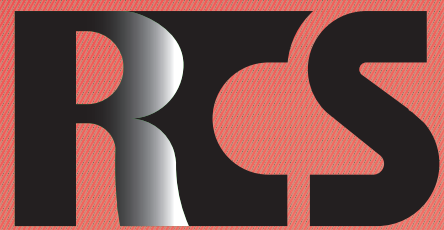

Depósito legal ppi $201502 Z U 4662$

Esta publicación científica en formato digital es continuidad de la revista impresa Depósito Legal: pp $197402 Z$ Z789

- ISSN: 1315-9518 • ISSN-E: 2477-9431

Revista de Ciencias Sociales

Universidad del Zulia. Revista de la Facultad de Ciencias Económicas y Sociales

Vol. XXVII,

No. Especial 3, 2021

Esta publicación científica en formato digital es continuidad de la revista impresa Depósito Legal: pp $197402 Z$ Z789

ISSN: $1315-9518$ 


\title{
Diversificación geográfica de las exportaciones de mango ecuatoriano*
}

\author{
Quiñónez Caicedo, Luisa Nicole ${ }^{* *}$ \\ Quiñónez Cabeza, Betty Maribel ${ }^{* * *}$ \\ Custode Quiñónez, Johanna Alejandra*** \\ Rodríguez Lara, John Jesús ${ }^{* * * * *}$
}

\section{Resumen}

La diversificación geográfica de las exportaciones permite a las empresas mitigar el riesgo al que están expuestas al exportar a un solo mercado, así como también a lograr economías de escalas y mayores ingresos. Esta investigación tiene como objetivo estudiar la diversificación geográfica de las exportaciones de mango ecuatoriano. La metodología de investigación se basó en un análisis empírico con alcance exploratorio y enfoque mixto, se realizó el cálculo del Índice Herfindahl-Hirschman durante el periodo 20162020, utilizando datos proporcionados por el Banco Central del Ecuador a nivel de subpartidas del Sistema Armonizado. Los resultados arrojaron que Ecuador exportó a cerca de 20 destinos en 2020, sin embargo, más del $80 \%$ de las exportaciones se destinaron hacia Estados Unidos, mientras que las exportaciones a los demás países fueron mínimas. Se concluyó, que la diversificación geográfica de las exportaciones de mango ecuatoriano se ve más afectada por factores relacionados a procedimientos nacionales y operatividad de las instituciones públicas inmersas en el comercio exterior, que no permiten un crecimiento constante y restan competitividad a las exportaciones.

Palabras clave: Diversificación geográfica; exportaciones; mango; sector exportador; Ecuador.

* Este documento es un producto generado a partir del proyecto de investigación intitulado: "Impacto Socioeconómico del Puerto Marítimo en la Provincia de Esmeraldas del periodo 2010 al 2017". Financiado por la Universidad Técnica de Esmeraldas Luis Vargas Torres, Ecuador.

** Magíster en Dirección del Comercio Exterior e Internacionalización de Empresas. Ingeniera en Comercio Exterior mención Negocios Internacionales. Investigadora Invitada en el proyecto Impacto Socioeconómico del Puerto Marítimo en la Provincia de Esmeraldas del periodo 2010 al 2017 de la Universidad Técnica de Esmeraldas Luis Vargas Torres, Ecuador. E-mail: nicolequinonezc@hotmail. com (iD ORCID: https://orcid.org/0000-0002-7290-8662

*** Magíster en Dirección y Administración de Empresas (MBA). Ingeniera Comercial. Docente de la Universidad Técnica de Esmeraldas Luis Vargas Torres, Ecuador. E-mail: betty.quinonez@utelvt.edu. ec (iD) ORCID: https://orcid.org/0000-0002-3964-2153

**** Magíster en Dirección del Comercio Exterior e Internacionalización de Empresas. Ingeniera en Comercio Exterior. Docente de la Universidad Técnica de Esmeraldas Luis Vargas Torres, Ecuador. E-mail: johanna.custode.quinonez@utelvt.edu.ec (iD) ORCID: https://orcid.org/0000-0002-0891-9359

***** Licenciado en Administración de Empresas con énfasis en Negocios Internacionales. E-mail: jirodriguezlara9@hotmail.com (D) ORCID: https://orcid.org/0000-0002-5406-6174 


\title{
Geographical diversification of Ecuadorian mango exports
}

\begin{abstract}
The geographical diversification of exports allows companies to mitigate the risk they are exposed to when exporting to a single market, as well as to achieve economies of scale and higher income. This research aims to study the geographical diversification of Ecuadorian mango exports. The research methodology was based on an empirical analysis with exploratory scope and a mixed approach, the Herfindahl-Hirschman Index was calculated during the 2016-2020 period, using data provided by the Central Bank of Ecuador at the level of sub-items of the Harmonized System. The results showed that Ecuador exported to about 20 destinations in 2020 , however, more than $80 \%$ of exports went to the United States, while exports to other countries were minimal. It was concluded that the geographical diversification of Ecuadorian mango exports is more affected by factors related to national procedures and the operation of public institutions involved in foreign trade, which do not allow constant growth and reduce the competitiveness of exports.
\end{abstract}

Keywords: Geographic diversification; exports; mango; export sector; Ecuador.

\section{Introducción}

La globalización ha llevado a una evidente aceleración de la economía mundial, generando oportunidades para las empresas y países que han sabido adaptarse a los rápidos y constantes cambios del entorno, aunque también creando desafíos y afectaciones para aquellos que no. Por cuanto, tal como lo señalan Proaño, et al. (2019), en conjunto con el proceso de industrialización en el ámbito mundial, la globalización "obliga a los mercados a ser cada vez más competitivos y eficiente, puesto que una de las características teóricas que tiene este paradigma es la apertura y unificación de todos los mercados a nivel mundial" (p.83).

Al respecto, Molero, et al. (2020) señalan que la globalizalización ha sido ampliamente estudiada desde muchos puntos de vistas y por varios autores desde mediados o finales de los años ochentas, encontrando que existen efectos positivos sobre el crecimiento de la economía por medio de la adjudicación de recursos (Samimi y Jenatabadi, 2014), desarrollo de tecnologías, aprovechamiento de los factores y mejoras en la productividad, así como también el aumento del capital; sin embargo, también hallaron efectos nocivos sobre economías con un marco institucional débil e inestabilidad política.

En este sentido, la globalización juega un papel importante para las empresas, pues permite reducir costos y mejorar la competitividad en precios y calidad de los bienes ofertados. Desde una mirada económica, tiene dos componentes: La globalización de mercados y la de la producción. Con la globalización de los mercados, las empresas ofrecen bienes estandarizados fuera del mercado nacional, es decir, en otros mercados internacionales, esto ayuda a la creación de un mercado global; por otra parte, la globalización de la produción, permite a las empresas hallar proveedores alrededor del mundo y aprovechar las ventajas que dejan las diferencias en precios y calidad de los factores de producción (Ristovska y Ristovska, 2014).

La globalización de los mercados, 
conduce a un proceso de internacionalización de las empresas a través de las exportaciones hacia nuevos mercados, la cual es entendida, como una estrategia llevada a cabo con el fin de incrementar las ventas, obtener mayores ingresos, reducir costos, alcanzar economías de escalas y mayor productividad, así como competitividad, mitigar el riesgo de sus operaciones y aprovechar las oportunidades que los mercados internacionales ofrecen (Sarmiento, 2014).

Desde la perspectiva económica, la diversificación de mercados es necesaria para las economías, contribuye al crecimiento y desarrollo a través de varios canales, entre estos: Aumentar las fuentes de ingresos y reducir los efectos adversos de la inestabilidad de las exportaciones en términos de intercambios comerciales (Osakwe, SantosPaulino y Dogan, 2018), así como también sufrir menores efectos negativos producto de los choques externos (Xuefeng y Yaşar, 2016).

La diversificación también es entendida como uno de los principales determinantes del crecimiento (Sannasse, Seetanah y Lamport, 2014). No obstante, aunque la diversificación de las exportaciones reviste de gran importacia para las economías, no todas han logrado tener la misma experiencia, pues los países desarrollados logran una mayor diversificación geográfica de las exportaciones que los países en desarrollo, aunque en las últimas décadas estos últimos han intentado penetrar en nuevos mercados (Vahalík, 2015).

En Ecuador, las exportaciones constituyen una de las principales fuentes de ingresos y generadoras de liquidez para la economía. La economía ecuatoriana depende de las exportaciones petroleras y productos primarios (Quinde-Rosales, et al., 2020), por lo que, se ha visto afectada en gran medida debido a las variaciones en los precios internacionales del petróleo (Proaño, et al., 2019). No obstante, las exportaciones no petroleras constituyen una oportunidad como alternativas para ampliar y diversificar las exportaciones y no depender altamente de las exportaciones petroleras.

En este sentido, las exportaciones de frutas tradicionales y en especial no tradicionales y hortalizas, se convierten en una opción importante, una de estas es el mango (Rodriguez, 2020). De acuerdo a cifras del Banco Central del Ecuador (BCE, 2021), el volumen de exportaciones de mango en 2020 fue de aproximadamente $49.160,4$ toneladas métricas y alrededor de $\$ 35$ millones de dólares FOB; sin embargo, del total de exportaciones, más del $80 \%$ fueron destinadas hacia Estados Unidos, al igual que en al menos los últimos 5 años, seguido de Colombia con $10,87 \%$, Canadá con $2,47 \%$, Holanda $1,05 \%$; mientras que a los demás destinos se exportó menos del $1 \%$.

La participación de Estados Unidos en el total de las exportaciones de mango, se ha mantenido en alrededor del $80 \%$ en los últimos años y los demás destinos continúan siendo los mismos, por lo que las exportaciones de mango no se han diversificado, no han logrado entrar, ni penetrar otros mercados.

La diversificación geográfica de las exportaciones de mango, figura beneficios para el sector exportador en Ecuador, por lo cual, este artículo tiene como objetivo estudiar la diversificación geográfica de las exportaciones de mango ecuatoriano. La metodología se basó en un análisis empírico con alcance exploratorio y enfoque mixto, realizando el cálculo del Indice HerfindahlHirschman (IHH) durante el periodo 20162020, con datos proporcionados por el Banco Central del Ecuador a nivel de subpartidas del Sistema Armonizado (SA); además, se indagó en el sector exportador del mango, con el fin de realizar entrevistas a empresas exportadoras y conocer la situación de las exportaciones.

Por otra parte, el alcance de la investigación permitió hallar información referente a los factores que influyen en la diversificación geográfica de las exportaciones de mango ecuatoriano, como los costos de transporte y logísticos, así como gubernamentales (Aguirre y Jaramillo, 2015). De igual manera, a través del enfoque mixto (Hernández, Fernández y Baptista, 2014), se manejó un proceso metodológico para la recopilación de los datos estadísticos e 
información a través de fuentes primarias y secundarias.

\section{Diversificación de las exportaciones}

La diversificación geográfica de las exportaciones, ha sido el centro de estudio en las últimas dos décadas, dada la importancia de ampliar la estructura exportadora de las economías, tanto en la oferta de productos como en el incremento del número de mercados de destino; esto porque, como Hinlo y Arranguez (2017) indican, las exportaciones en un número reducido de mercados llevan a los países a sufrir desde vulnerabilidades a inestabilidades futuras en el mercado nacional; al contrario, al reducir la dependencia, podrían mitigar riesgos futuros como riesgos económicos dados por la volatilidad e inestabilidad de las divisas obtenidas, que además, tienen efectos sobre el crecimiento, el empleo, la planificación de inversiones, así como en la capacidad de importación y exportación.

Se ha planteado que un aumento en la diversificación de las exportaciones de productos como de destinos, influye en el crecimiento económico de los países. Desde el punto de vista económico, esta relación podría ser cierta, dado que las exportaciones son un rubro primordial en el Producto Interno Bruto (PIB) de una economía, por lo tanto, es propio señalar que un incremento en las exportaciones reflejaría un aumento en el PIB (Agosin, 2009). En este sentido, las economías con una oferta exportable diversificada reflejan mayor PIB, con la particularidad de que registran un crecimiento económico con tendencia progresiva, en comparación con los países con una oferta exportable no diversificada.

Vázquez y Morales (2017) sostienen que se debe considerar los efectos dinámicos de la diversificación de las exportaciones, debido a que el crecimiento a largo plazo se relaciona enormemente con la producción y así extender la oferta exportable de bienes. De esta manera, las economías que poseen pocas fuentes para el aumento de la productividad, la fuente mayor de inversión para avances respecto a la diversificación de su cartera comercial, procede de capitales del extranjero, puesto que los bienes de capital nuevos representan un cambio en la producción, con esto la apertura de nuevos sectores donde la productividad de los factores es superior a la de los sectores existentes.

Al respecto, Ghani, Mahmood y Din (2012) llevaron a cabo un estudio exploratorio para analizar los determinantes de la diversificación de mercados a nivel de empresas, utilizando el marco de regresión logística. Este análisis se basó en la encuesta del estudio "Desafíos relacionados con el comercio que enfrentan los exportadores en Pakistán", realizado por el Instituto de Economía del Desarrollo de Pakistán (PIDE) en conjunto con la Organización de las Naciones Unidas para el Desarrollo Industrial (ONUDI), la encuesta consideró una muestra de 157 empresas de las provincias de Sindh y Punjab, cuya actividad principal es la fabricación de bienes de exportación.

En ese sentido, las variables consideradas fueron: Mercados de exportación, estructura de propiedad, tamaño de la empresa y ubicación. Los resultados arrojaron que la experiencia acumulada de las compañías más antiguas, les otorga mayores probabilidades de aprovechar las oportunidades y cumplir con los requisitos para entrar a nuevos mercados internacionales, además que, los factores que inciden positivamente en la diversificación, son el tamaño y la productividad laboral, acompañados de economías de escala y costos competitivos. No obstrante, señalan que las políticas fijadas para promocionar las exportaciones y la diversificación de mercados, deben estar encaminadas a establecer un clima adecuado para promover la inversión privada y la expasión empresarial.

Por su parte, Cos, Colom y Cabasés (2018) realizaron un estudio para analizar los factores de las empresas que eligen una estrategia de diversificación de exportaciones o mercados y cómo ésta ayuda en el desempeño exportador, utilizando una muestra de empresas exportadoras españolas con una 
metodología de regresión logística binomial (para las hipótesis 1-4) y la prueba de White para probar la última hipótesis (H5). El estudio permitió lograr un modelo que agrupe las variables determinantes en el desempeño exportador de las empresas y la estrategia de diversificación de mercados, y establecer un vínculo entre esta estrategia y el éxito de las exportaciones.

Concluyendo este estudio, que el compromiso internacional, el tamaño de la empresa, la edad y el nivel de propiedad extranjera en el capital social de la empresa, son los factores que más influyen en la estrategia de diversificación; sin embargo, a diferencia de otros autores, sostienen que la inversión en innovación, el sector involucrado y los gastos en actividades de comunicación como porcentaje de las ventas, también influyen. Así mismo, hallaron que el desempeño exportador de cada empresa difiere por sus tamaños, siendo las empresas grandes o con más experiencia las que adquieren un grado mayor de diversificación.

Por otra parte, Shepherd (2010) realizó uno de los primeros estudios sobre los factores que impulsan la expansión geográfica de comercio de países en desarrollo, a través de un modelo de Poisson IV, tomando una muestra de 123 países en desarrollo. El estudio demostró, que los costos de exportación, aranceles y costos de transporte internacional, son determinantes importantes de la diversificación geográfica de las exportaciones. Halló que un $10 \%$ en la reducción de los costos de exportación y transporte, están asociados aproximadamente con un aumento del $6 \%$ en el número de mercados de exportación; mientras que una reducción del $10 \%$ de aranceles se relaciona con un aumento del 5\% de la diversificación geográfica de las exportaciones.

Además, el estudio arrojó que la diversificación geográfica de las exportaciones en los países pobres, sufren un efecto más fuerte debido a los costos de exportación y, que los sectores que están relativamente más diferenciados, se ven afectados mayormente por los costos de exportación y los de transporte, esto según explica, porque por lo general la diferenciación se asocia con un mayor valor agregado de fabricación.

La diversificación geográfica, se ha estudiado ampliamente en las últimas dos décadas, la importancia de la diversificación como un impulso al crecimiento económico ha llevado a una tendencia creciente en apostar por entrar a nuevos mercados, en especial en los países en desarrollo. Amurgo-Pacheco y Pierola (2008), estudiaron los patrones de diversificación de las exportaciones en los países en desarrollo, con relación a los márgenes intensivos y extensivos del comercio, encontrando que la diversificación geográfica muestra más dinamismo que la diversificación por productos. El estudio también señala, que los costos comerciales como de transporte $\mathrm{y}$ acuerdos comerciales, tienen un impacto significacitivo en los márgenes intensivos.

Por lo tanto, existiría una causalidad entre la diversificación de las exportaciones y el crecimiento, que puede representar un gran incentivo para aumentar el crecimiento de la economía. Dicho de otra manera, las economías que no cuentan con una oferta exportable diversificada, tanto en bienes como destinos, no lograrán extender su ventaja comercial y estarán más expuestas a los choques externos. Las exportaciones representan un importante rubro en la economía ecuatoriana, la política comercial busca ampliar la oferta exportable y romper con la dependencia de las exportaciones petroleras y tradicionales, además de incrementar los destinos de estas (Quiñónez y Quiñónez, 2020); por lo cual, siendo el mango parte del rubro de las exportaciones no tradicionales, se deben estudiar los factores que inciden en su diversificación geográfica.

\section{Exportación de mango ecuatoriano}

La producción de mango en Ecuador para su comercialización data de 1980, debido a la demanda exigida por los países industrializados y de la falta de capacidad de los grandes exportadores de la época para atender dicha demanda (en especial de los países de Asia). Luego de su introducción en 
el mercado mundial en la década de los 90's, el mango ecuatoriano ha continuado como un referente para las importaciones de los principales países, por lo que el $84 \%$ de la producción es destinada a exportación.

Durante la última década, Ecuador ha estado entre los 20 principales países exportadores de mango a nivel mundial. En 2019, se ubicó en la posición número
16 y representó aproximadamente el 1,2\% de la participación mundial; sin embargo, en los últimos 5 años más del $80 \%$ de las exportaciones se han destinado al mercado estadounidense, a excepción de Colombia y Países Bajos, los demás países representaron menos del $1 \%$ de participación en las exportaciones ecuatorianas (ver Gráfico I).

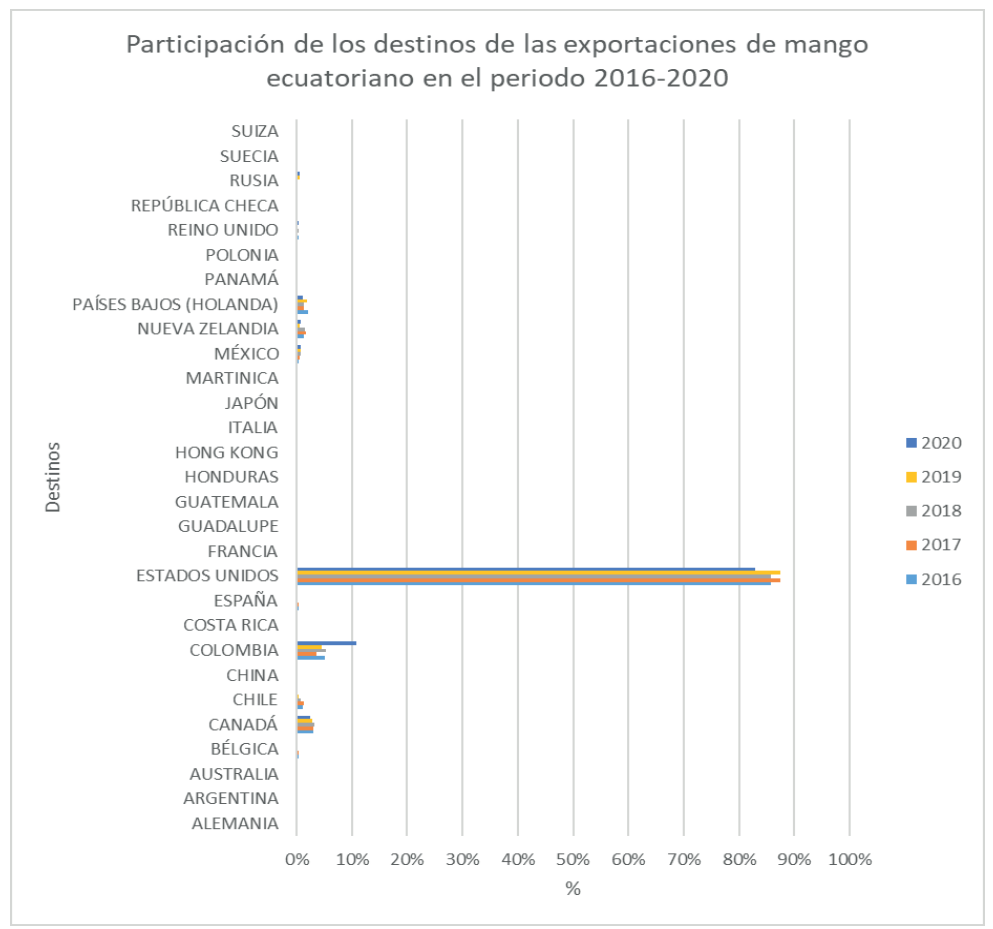

Fuente: Banco Central del Ecuador (2021).

\section{Gráfico I: Participación de los destinos de las exportaciones de mango ecuatoriano periodo 2016-2020}

El Índice de Herfindahl Hirschman (IHH), es utilizado en economía industrial para medir el grado de concentración o diversificación de los mercados. Este índice es calculado a través de la siguiente ecuación: 


$$
I H H=\sum_{i=1}^{n}\left(\frac{p_{i}}{P}\right)^{2}
$$

Donde: $\mathrm{P}$ es igual a las exportaciones totales de un país; $p i$ es igual a las exportaciones al país $\boldsymbol{i} ; \boldsymbol{n}$ es el número total de países. La suma de los cuadrados de las participaciones de las exportaciones de cada país en el total de las exportaciones del país analizado se conoce como Índice de Herfindahl Hirschman.

Un resultado del indicador $\mathrm{IHH}<1,000$ muestra un Mercado Diversificado; mientras que entre $1,000<\mathrm{IHH}<1,800$ un Mercado Moderadamente Diversificado; $\mathrm{IHH}>1,800$ un Mercado Concentrado; y, $\mathrm{IHH}=10,000$ un Monopolio. Sin embargo, debido a que su rango varía dependiendo de la información con la que se cuenta, se utiliza el Índice de Herfindahl-Hirschman Normalizado (IHHN), que no es más que una normalización del rango que varía entre 0 y 1 ; así, un $\mathrm{IHH}<0.1$ representa un Mercado Diversificado; entre
$0.1<\mathrm{IHH}<0.18$ un Mercado Moderadamente Diversificado; un $\mathrm{IHH}>0.18$ un Mercado Concentrado, mientras que un $\mathrm{IHH}=1$ un Monopolio. El IHHN se calcula de la siguiente manera:

$$
I H N=\frac{\sum_{i=1}^{n}\left(\frac{p_{i}}{P}\right)^{2}-\frac{1}{n}}{1-\frac{1}{n}}
$$

Este indicador es uno de los más utilizados en economía para medir el grado de concentración o diversificación de las exportaciones, dado esto, se calculó este índice para medir el grado de diversificación geográfica de las exportaciones de mango ecuatoriano para el periodo 2016-2020, tomando datos proporcionados por el Banco Central del Ecuador, correspondientes al valor total de las exportaciones por destinos de la subpartida arancelaria 0804502010 durante el periodo señalado (ver Tabla 1).

Tabla 1

Índice de Herfindahl-Hirschman

\begin{tabular}{ccccc}
\hline 2016 & 2017 & 2018 & 2019 & 2020 \\
\hline 0,7 & 0,8 & 0,7 & 0,8 & 0,7 \\
\hline
\end{tabular}

Fuente: Banco Central del Ecuador (2021).

Como muestran la Tabla 1 y Gráfico II, las exportaciones de mango ecuatoriano se encontraron altamente concentradas con un índice mayor o igual a 0,7 durante el periodo analizado. El IHHN fue de 0,7 en 2016; 0,8 en 2017; 0,7 en 2018; 0,8 en 2019; y, 0,7 en 2020 (ver Tabla 1). Estos resultados son explicados al analizar el Gráfico II, que muestra la participación de los destinos de las exportaciones de mango ecuatoriano durante el periodo analizado, donde más del $80 \%$ de las exportaciones se destinaron al mercado estadounidense. 


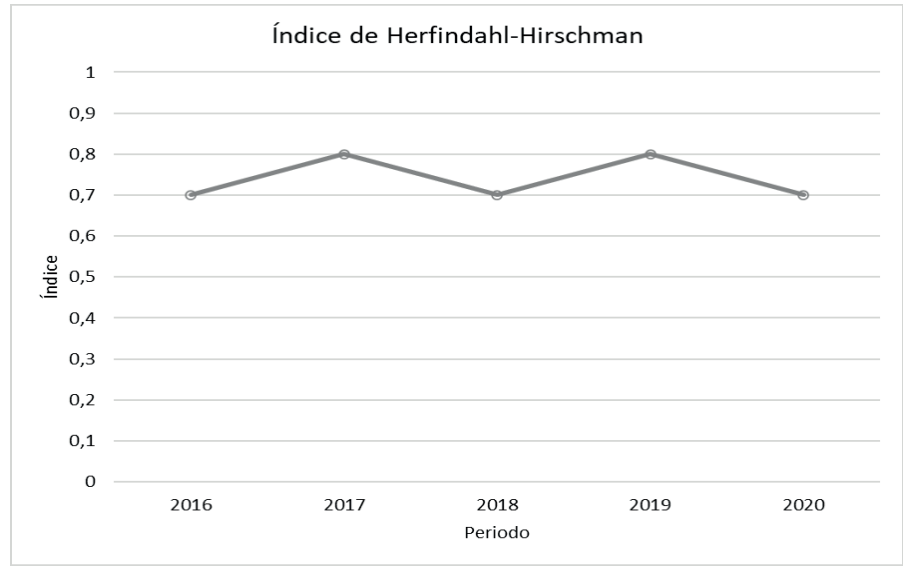

Fuente: Banco Central del Ecuador (2021).

Gráfico II: Índice de Herfindahl-Hirschman

\section{Factores que influyen en las exportaciones de mango ecuatoriano}

La diversificación geográfica de las exportaciones de mango ecuatoriano, se ve afectada por varios factores como la falta de gestión por parte de las autoridades gubernamentales, para cumplir con los requisitos solicitados por las autoridades de otros países, como en el caso de las exportaciones al mercado chileno, que en 2020 no se logró exportar a este destino, mientras que en años anteriores se exportaba entre 20 a 25 contenedores. La apreciación del dólar frente a otras monedas, también influye en la diversificación y resta competitividad en precios.

Otros factores están relacionados con el despacho de los contenderos y las instituciones del Estado. Uno de los casos más comunes se da con la policía antinarcóticos, que solicita realizar inspección física de los contenedores; sin embargo, esto no es posible porque los contenedores de frutas, en especial los destinados al mercado estadounidense, deben llevar un sello colocado por un funcionario del Servicio de Inspección Sanitaria de Plantas y Animales (APHIS) del Departamento de Agricultura de los Estados Unidos, a través del ARCSA, debido a un convenio entre el APHIS, ARCSA y la Fundación Mango Ecuador.

Entre los términos, se indican que, el contenedor no puede ser abierto en el puerto de origen luego que el sello es colocado, caso contrario no será recibido en el puerto de destino; pese a estas indicaciones por parte de los exportadores y Fundación Mango Ecuador, cada año abren entre uno o dos. Así mismo, otro de los factores se relaciona con el tiempo que toma obtener permisos y requisitos en las instituciones públicas.

Por otra parte, los factores logísticos también afectan las exportaciones, debido a que las empresas reciben los contenedores sucios y no pueden aceptarlos o las navieras los envían tarde, esto retrasa la logística de las empresas, que deben enviar los contenedores máximo 12 horas antes de que el barco inicie a ser cargado. Así mismo, la falta de acuerdos comerciales con socios comerciales clave 
como Estados Unidos y México, afecta a las exportaciones; en el caso de Estados Unidos, el mango se ve beneficiado con la reducción arancelaria del 0\% debido al Sistema General de Preferencias (SGP), sin embargo, este venció el 31 de diciembre de 2020; pero con relación a México, el mango ecuatoriano ingresa con un arancel entre el 12 y $13 \%$.

De igual manera, en cuanto al transporte, se encontró que, no hay rutas aéreas frecuentes a destinos del mercado asiático, a través de la cual es mayormente conveniente transportar el mango, por el tiempo de traslado; sin embargo, el costo es muy elevado por la falta de frecuencias, lo que obliga a las empresas a continuar exportando a los mercados tradicionales como Estados Unidos.

\section{Conclusiones}

Las exportaciones de mango, representan una importante oportunidad para diversificar la oferta exportable ecuatoriana, generar ingresos provenientes de productos no petroleros y no tradicionales e irse apartando de la dependencia de los ingresos provenientes del petróleo. Este artículo tuvo como objetivo estudiar la diversificación geográfica de las exportaciones de mango ecuatoriano, así, el cálculo del Índice de Herfindahl Hirschman de las exportaciones de mango ecuatoriano durante el periodo 2016-2020, mostró que las exportaciones se encuentran altamente concentradas en un solo mercado, el resto de los mercados, a excepción de Colombia y Países Bajos, representaron una participación mínima.

Asimismo, se halló que las exportaciones se ven afectadas por problemas logísticos, la falta de acuerdos comerciales, la falta de frecuencias de las navieras hacia otros destinos, los costos altos del transporte, pero principalmente, por factores que se relacionan con instituciones del sector público inmerso en el comercio exterior, como la Policía Antinarcóticos y, la gestión pública del Ecuador, que no permiten un crecimiento constante y restan competitividad a las exportaciones.

Finalmente, es imperioso la coordinación entre las autoridades del Estado, los exportadores y gremios que intervienen en las exportaciones de esta fruta, que representa un rubo significativo en las exportaciones de frutas no tradicionales para el Ecuador, de manera que se sociabilicen los problemas que enfrentan al ingresar a nuevos mercados, a fin de gestionar, así como agilitar las negociaciones internacionales, beneficiando al sector exportador del mango y a la economía nacional.

\section{Referencias bibliográficas}

Agosin, M. R. (2009). Crecimiento y diversificación de las exportaciones en economías emergentes. Revista Cepal, (97), 117-134. https:// repositorio.cepal.org/bitstream/ handle/11362/11274/097117134 es.pdf?sequence $=1 \&$ is Allowed $=y$

Aguirre, J. C., y Jaramillo, L. G. (2015). El papel de la descripción en la investigación cualitativa. Cinta de Moebio, (53), 175-189. https://doi.org/10.4067/ S0717-554X2015000200006

Amurgo-Pacheco, A., y Pierola, M. D. (2008). Patterns of Export Diversification in Developing Countries: Intensive and Extensive Margins. Policy Research Working Paper (4473). https://openknowledge. worldbank.org/bitstream/ handle/10986/6447/wps4473. pdf? sequence $=1 \&$ isAllowed $=y$

Banco Central del Ecuador - BCE (2021). Comercio Exterior. Estadísticas de Exportación de Bienes: Exportaciones por Subpartida y País Destino. BCE. https://www.bce.fin.ec/index.php/ comercio-exterior

Cos, P., Colom, A., y Cabasés, Á. (2018). Geographic export diversification: 
Determinants and their link with export performance. Thunderbird International Business Review, 61(2), 397-411. https://doi.org/10.1002/ tie. 21991

Ghani, E., Mahmood, T., y Din, M-U. (2012). Market diversification and firms' characteristics of export-oriented manufacturers in Pakistan. The Pakistan Development Review, 51(2), 103-115.

Hernández, R., Fernández, C., y Baptista, P. (2014). Metodología de la investigación. McGraw-Hill.

Hinlo, J. E., y Arranguez, G. I. S. (2017). Export Geographical Diversification and Economic Growth Among ASEAN Countries. MPRA Paper, (81333). https://mpra.ub.uni-muenchen.de/id/ eprint $/ 81333$

Molero, L. E., Anchundia, J. C., Patiño, R. J., y Escobar, Y. M. (2020). Crecimiento económico y apertura comercial: Teoría, datos y evidencia (19602017). Revista de Ciencias Sociales (Ve), XXVI(4), 476-496. https://doi. org/10.31876/rcs.v26i4.34675

Osakwe, P. N., Santos-Paulino, A. U., y Dogan, B. (2018). Trade dependence, liberalization, and exports diversification in developing countries. Journal of African Trade, 5(1-2), 19-34. $\quad$ https://doi.org/10.1016/j. joat.2018.09.001

Proaño, S. A., Quiñonez, E. S., Molina, C. J., y Mejía, O. G. (2019). Desarrollo económico local en Ecuador: Relación entre producto interno bruto y sectores económicos. Revista de Ciencias Sociales (Ve), XXV(1), 82-98. https:// doi.org/10.31876/rcs.v25i1.29598

Quinde-Rosales, V., Bucaram-Leverone, R., Saldaña-Vargas, M., y DiazCampo, C. (2020). Relación entre los ingresos petroleros y el gasto público ecuatoriano. Revista de Ciencias Sociales (Ve), XXVI(3), 118129. https://doi.org/10.31876/rcs. v26i3.33237

Quiñónez, M. R., y Quiñónez, L. N. (2020). Política de comercio exterior en Ecuador: Un análisis comparativo. Centro Sur. Social Science Journal, 4(2), 266-284. http://www. centrosureditorial.com/index.php/ revista/article/view/81/214

Ristovska, K., y Ristovska, A. (2014). The Impact of Globalization on the Business. Economic Analysis, Instituto de Ciencias Económicas, 47(3-4), 8389.

Rodriguez, J. (2020). Factores que influyen en la exportación de mango ecuatoriano a nuevos mercados (Proyecto de investigación de pregrado). Universidad Ecotec, Guayaquil, Ecuador.

Samimi, P., y Jenatabadi, H. S. (2014). Globalization and Economic Growth: Empirical Evidence on the Role of Complementarities. PLoS ONE, 9(4), e87824. $\quad \underline{\text { https://doi.org/10.1371/ }}$ journal.pone.0087824

Sannasse, R. V., Seetanah, B., y Lamport, M. J. (2014). Diversificación de las exportaciones y crecimiento económico: el caso de Mauricio. En M. Jansen, M. S. Jallab y M. Smeets (Comps.), Conectarse a los mercados mundiales. Retos y oportunidades: Estudios de casos presentados por titulares de cátedras OMC (pp. 11-25). Organización Mundial del Comercio.

Sarmiento, S. (2014). Estrategias de internacionalización y globales para países en desarrollo y emergentes. Dimensión Empresarial, 12(1), 111138.

Shepherd, B. P. (2010). Geographical diversification of developing country 
Diversificación geográfica de las exportaciones de mango ecuatoriano

Quiñónez Caicedo, Luisa Nicole; Quiñónez Cabeza, Betty Maribel;

Custode Quiñónez, Johanna Alejandra y Rodríguez Lara, John Jesús

exports. World Development, 38(9), 1217-1228. https://doi.org/10.1016/j. worlddev.2010.02.005

Vahalík, B. (2015). Analysis of export diversification development of the European Union and BRICS countries. Ekonomická Revue - Central European Review of Economic Issues, 18, 59-69.

Vázquez, R., y Morales, R. A. (2017). Diversificación de las exportaciones y competitividad externa en la industria. Hacia la construcción de una tipología para el caso de países de ingresos medios. Cuadernos de Economía, 40(114), 208-222. https:// doi.org/10.1016/j.cesjef.2016.09.002

Xuefeng, Q., y Yaşar, M. (2016). Export Market Diversification and Firm Productivity: Evidence from a Large Developing Country. World Development, 82, 28-47. https://doi. org/10.1016/j.worlddev.2016.01.017 